\title{
Euritrematose bovina
}

\section{Bovine eurytrematosis}

\section{Clóvis Antonio Bassani ${ }^{1 *}$; Luís Antonio Sangioni²; João Paulo Elsen Saut ${ }^{3}$; Selwin Arlington Headley ${ }^{4}$; Milton Hissashi Yamamura ${ }^{5}$}

\section{Resumo}

Nesta revisão de literatura sobre os trematódeos do gênero Eurytrema (LOOSS, 1907), pertencentes à família Dicrocoeliidae (ODHNER, 1910), são abordados aspectos como: espécies, morfologia, hospedeiros, distribuição geográfica, patogenia e patologia, diagnóstico, tratamento e controle terapêutico.

Palavras-chave: Eurytrema, trematoda, revisão, bovino, pâncreas

\begin{abstract}
In this review about the trematoda of genre Eurytrema (LOOSS, 1907) that belong to Dicrocoeliidae family (ODHNER, 1910), aspects as species, morphology, host, geographic distribution, pathogenesis and pathology, diagnosis and therapeutic control are boarded.
\end{abstract}

Key words: Eurytrema, trematoda, review, cattle, pancreas

\section{Introdução}

Um dos problemas enfrentados na pecuária de corte e leite, são as endo e ectoparasitoses, que interferem no desenvolvimento e no rendimento dos animais, causando acentuados prejuízos em toda a sua cadeia produtiva.

As espécies do gênero Eurytrema (LOOSS, 1907), chamam a atenção pelos índices que são observados em pâncreas de bovinos abatidos em matadouros frigoríficos nos diversos estados brasileiros (BRANT, 1962; AZEVEDO et al., 2000;
Destas espécies se destacam: Eurytrema pancreaticum (JANSON, 1889) e Eurytrema coelomaticum (GIARD; BILLET, 1892) que são parasitas comuns de ductos pancreáticos, ocasionalmente ductos biliares e raramente intestino delgado de ruminantes. O primeiro tem sido descrito em bovinos, caprinos, ovinos, bubalinos, suínos, camelídeos, cervídeos e homem, enquanto que o segundo ocorre em bovinos, caprinos, ovinos, bubalinos, leporinos e camelídeos (MATTOS JÚNIOR; VIANNA, 1987). YAMAMURA, 2005).

1 Médico Veterinário, Mestre. Docente da disciplina de Higiene e Inspeção dos Produtos de Origem Animal. Faculdade Integrado de Campo Mourão- PR. Endereço: Av. José Custódio de Oliveira, 435 - Centro. Campo Mourão / PR. CEP: 87301-020 - Fone (44) 35252169. e-mail clovisbassani@yahoo.com.br

2 Médico Veterinário, Doutor. Docente da Disciplina de Parasitologia e Doenças Parasitárias. Faculdade Integrado de Campo Mourão- PR.

3 Médico Veterinário, Mestre. Docente da Disciplina de Clínica de Grandes Animais. Faculdade Integrado de Campo Mourão- PR.

4 Médico Veterinário, Doutor Docente do Departamento de Medicina Veterinária Preventiva/CCA da Universidade Estadual de Londrina- PR.

5 Médico Veterinário, Doutor. Professor Titular do Departamento de Medicina Veterinária Preventiva/CCA da Universidade Estadual de Londrina- PR.

* Autor para correspondência 
Dada a grande semelhança morfológica entre as duas espécies de parasitas citadas, alguns pesquisadores as registraram como sinônimas. Entretanto, de acordo com Travassos (1944) a espécie que ocorre no Brasil é o E. coelomaticum.

Os autores, relatam quase sempre, os aspectos parasitológicos, histopatológicos e descrições de necropsias, ocultando as informações referentes à patologia clínica em animais severamente infectados (PALATKIN, 1978; BRITO; FRANCIS; CARVALHO, 1981; BORAY, 1985; GASTE, 1991; BELÉM; OLIVEIRA; PADOVANI, 1994).

$\mathrm{Na}$ China e no Japão, nas últimas décadas, essa parasitose também tem sido motivo de preocupações dos pesquisadores, porque a mortalidade em bovinos vem aumentando anualmente (SHIEN; LIU; HUANG, 1979; SAKAMOTO et al., 1984).

Vários pesquisadores comprovaram a ineficiência dos produtos anti-helmínticos usados para combater a euritrematose em bovinos. Essa informação vem confirmar os resultados negativos que se obtêm quando se tenta tratar os animais parasitados com os produtos disponíveis (MATTOS JÚNIOR; VIANNA, 1987; YAMAMURA, 1989; ARAÚJO; BELÉM, 1993).

Algumas pesquisas revelam aspectos importantes da euritrematose bovina, sendo que, carecem ainda de maiores estudos, em face dos prejuízos causados à indústria extrativa de insulina devido às condenações sumárias de pâncreas parasitadas, já relatadas por (PALATKIN, 1978; BRITO; FRANCIS; CARVALHO, 1981; ZIEGLER et al., 1984; BORAY, 1985). Provavelmente ocorram também perdas econômicas quanto á produção de carne e leite, conforme afirmaram (BURGGRAAF, 1935; BASCH, 1966). Diversos autores (CÔDO, 1952; HARRIS, 1952; NIEBERLE; COHRS, 1970; MANNINGER; MOCSY, 1973; FREITAS, 1977; SAKAMOTO et al., 1980; KONO et al., 1980; SOULSBY, 1982; CORREA, 1983; CORREA et al., 1984; BORAY, 1985; BELÉM; SILVA; VIEIRA, 1986), encontraram em áreas endêmicas animais severamente infectados, demonstrando más condições nutricionais e inclusive, foi apontada por Correa et al. (1984), como responsável pelo elevado índice de mortalidade, atingindo 6,6\%, (19/300).

De acordo com Yamamura (1989), as lesões macroscópicas foram classificadas em três tipos, em função da extensão das lesões produzidas por $E$. coelomaticum. Tipo I - Ausência de lesões macroscópicas nos ductos e parênquima. Tipo II Presença de lesões macroscópicas somente nos ductos. Tipo III - Presença de lesões macroscópicas nos ductos e parênquima. Os estudos histológicos dessa infecção demonstram invariavelmente a ocorrência de pancreatite intersticial crônica, com o tecido glandular sendo substituído por tecido fibroso, dilatação e obstrução de ductos pancreáticos, focos de células gigantes do tipo corpo estranho e acúmulo de ovos do parasita tanto nos ductos quanto no parênquima do órgão.

Esta parasitose geralmente se manifesta sob a forma subclínica, não obstante, foram observados os seguintes sinais clínicos: emaciação e caquexia (CÔDO, 1952; MARTIN, 1972; SAKAMOTO et al., 1980; CORREA et al., 1984; BELÉM; SILVA; VIEIRA, 1986; MATTOS JÚNIOR., VIANNA, 1987), anorexia (KONO et al., 1980; SAKAMOTO et al., 1980), salivação espumosa (HARADA; WATO; FUJIWARA, 1980; SAKAMOTO et al., 1980; BORAY, 1985). A glicosúria foi relatada por (HORTA, 1918; HARRIS, 1952; HARADA; WATO; FUJIWARA, 1980; BELÉM; SILVA; VIEIRA, 1986); hiperglicemia (HARADA; WATO; FUJIWARA, 1980; SAKAMOTO et al., 1980), sendo que para Burggraaf (1935) a hiperglicemia é um achado inconstante. Níveis normais de amilase sérica foram encontrados por Sakamoto et al. (1980); diminuição e até ausência das enzimas como a amilase e a tripsina no suco pancreático foi verificado por Burggraaf (1935), proteína total normal ou com ligeiro aumento, amilase sérica diminuída, colesterol aumentado, relação albumina/globulina normal, G.O.T. (Transaminase Glutâmica Oxalacética) e G.P.T. (Transaminase Gutâmica Pirúvica) normais foram encontrados por Harada, Wato e Fujiwara, 
(1980). Como se pode notar, um diagnóstico clínico ou laboratorial preciso, torna-se extremamente difícil e, não raro, é feito somente pela necropsia do animal.

Esta endoparasitose, que causa lesões preponderantemente caracterizadas por pancreatite intersticial fibrosante e obliteração total ou parcial dos ductos pancreáticos, pode ocasionar transtornos, em graus variados, nas funções secretoras do pâncreas como foram relatados por (BURGGRAAF, 1935; SEIFRIED 1936; CÔDO, 1952; FOX et al. 1981).

Neste contexto, é sensato admitir que os processos digestivos e metabólicos dependentes das funções pancreáticas podem estar alterados e os animais portadores de lesões pancreáticas do tipo II e III, segundo Yamamura (1989), podem apresentar diminuição na digestibilidade e conseqüentemente, na assimilação dos alimentos. Perdas de elementos protéicos e lipídicos pelas fezes poderão ocorrer e refletir em alterações séricas, hematológicas e fecais muito antes dos animais demonstrarem sinais clínicos aparentes. (GASTE, 1991).

\section{Espécies}

Na classificação sistemática dos trematódeos de localização pancreática, ao longo dos anos surgiram dúvidas e quase todos os trabalhos inicialmente, descreviam os parasitos pela sua localização habitual nos órgãos, pela localização errática nos seus hospedeiros, seguida pelas características fenotípicas, através das descrições morfológicas das estruturas dos órgãos do parasito e por fim pelas análises citológicas dos cromossomos do trematódeo.

Looss e Cuffey (1907), revendo a nomenclatura taxonômica dos trematódeos digenéticos descritos por diversos autores salientaram que a medida e a posição relativa das ventosas representavam características específicas de E. coelomaticum, ou seja, o diâmetro da ventosa oral tinha a mesma medida do diâmetro da ventosa ventral e a distância entre as duas ventosas era menor em E. coelomaticum, quando comparado com E. pancreaticum. Também era menor a formação de grupos de folículos da glândula vitelogênica encontrada em E. coelomaticum. A partir destas considerações, outros autores começaram a caracterizá-los como espécies distintas e acrescentaram outras estruturas, como o útero, que em E. coelomaticum não forma curvas anteriores a ventosa ventral, e as glândulas vitelogênicas que são constituídos de pequenos folículos arredondados, tais observações foram realizadas por Eduardo, Manuel e Tongson (1976).

Travassos (1944), na revisão da família Dicrocoeliidae reconheceu efetivamente o $E$. coelomaticum, como espécie distinta e ressaltou ainda que a espécie prevalente na América do Sul era E. coelomaticum. Muitos autores a denominaram em seus trabalhos como E. pancreaticum, como Horta (1918), Freire (1967), Campos et al. (1974), Ribeiro et al. (1980), Busetti et al. (1983) e Correa et al, (1984), devendo na realidade, se tratar de $E$. coelomaticum levando em consideração a afirmação feita por Travassos (1944). O mesmo deve acontecer em outros países do continente sul americano, como a Venezuela, onde Mayaudon (1954) reportou a ocorrência de E. pancreaticum e levantou suspeita de que o animal parasitado fora importado do Brasil.

Tang e Tang (1977), realizaram trabalhos que diferenciaram os aspectos morfológicos dos parasitos adultos, bem como a morfologia das formas larvais encontradas em hospedeiros intermediários, ressaltando a segunda geração de esporocisto.

Moriyama, Tsuji e Seto (1980) estudaram paralelamente as características fenotípicas e genotípicas, através das análises cariológicas de cromossomos de tecido gonadal de E. coelomaticum e E. pancreaticum, designando-os neste estudo de tipo I e tipo II respectivamente e concluiu haver diferenças morfológicas e biométricas dos cromossomos, bem como as posições dos centrômeros destas duas espécies.

Vários estudos revelam que um grande número de autores ao longo dos anos vem se dedicando ao estudo do Eurytrema spp. contemplando os hospedeiros, a procedência e o tipo de infecção (Tabela I). 
Tabela 1. Principais publicações contemplando os autores, espécies, regiões de procedência e tipos de infecções realizadas, para o gênero Eurytrema spp.

\begin{tabular}{|c|c|c|}
\hline Autor (es) & Procedência & Tipo de Infecção \\
\hline \multicolumn{3}{|l|}{ Eurytrema coelomaticum } \\
\hline \multicolumn{3}{|l|}{ Bovino } \\
\hline Giard; Billet (1892) & Tokin & Natural \\
\hline Horta (1918) & Brasil & Natural \\
\hline Travassos (1918) & Brasil & Natural \\
\hline Figueiredo (1928) & Brasil & Natural \\
\hline Pinto; Almeida (1935) & Brasil & Natural \\
\hline Torres; Pinto (1935) & Brasil & Natural \\
\hline Carvalho (1944) & Brasil & Natural \\
\hline Travassos (1944) & Brasil & Natural \\
\hline Côdo (1952) & Brasil & Natural \\
\hline Watanabe (1960) & Japão & Natural \\
\hline Brant; Costa (1963) & Brasil & Natural \\
\hline Nosaka et al. (1970) & Japão & Natural \\
\hline Campo et al. (1974) & Brasil & Natural \\
\hline Molfi (1976) & Brasil & Natural \\
\hline Eduardo et al. (1976) & Filipinas & Natural \\
\hline Tang; Tang (1977) & China & Natural \\
\hline Shien et al. (1978) & China & Natural \\
\hline Kono et al. (1980) & Japão & Natural \\
\hline Moriyama et al. (1980) & Japão & Natural \\
\hline Souza; Rabelo (1981) & Brasil & Natural \\
\hline Yamamura et al. (1982) & Brasil & Natural \\
\hline Mattos JR (1987) & Brasil & Natural \\
\hline \multicolumn{3}{|l|}{ Caprino } \\
\hline Proença (1935) & Brasil & Natural \\
\hline Travassos (1944) & Brasil & Natural \\
\hline Tang; Tang (1977) & China & Experimental \\
\hline Sakamoto et al (1980) & Japão & Experimental \\
\hline Kono et al. (1986) & Japão & Experimental \\
\hline Mattos JR (1987) & Brasil & Natural \\
\hline \multicolumn{3}{|l|}{ Lebre } \\
\hline Sakamoto (1981) & Japão & Natural \\
\hline \multicolumn{3}{|c|}{ Camundongo Balb c/ nude+mouse } \\
\hline Sakamoto et al. (1981) & Japão & Experimental \\
\hline \multicolumn{3}{|l|}{ Gato } \\
\hline Chinone et al (1984) & Japão & Experimental \\
\hline \multicolumn{3}{|l|}{ Ovino } \\
\hline Carvalho (1940) & Brasil & Natural \\
\hline Travassos (1944) & Brasil & Natural \\
\hline Tang; Tang (1977) & China & Natural \\
\hline \multicolumn{3}{|l|}{ Bubalinos } \\
\hline Yamamura et al (1982) & Brasil & Natural \\
\hline \multicolumn{3}{|l|}{ Eurytrema pancreaticum } \\
\hline Janson (1889) & Japão & Natural \\
\hline Purvis (1931) & Malásia & Natural \\
\hline Travassos (1944) & Brasil & Natural \\
\hline Florence (1939) & Madagascar & Natural \\
\hline Tang (1950) & China & Natural \\
\hline Mayaudon (1954) & Venezuela & Natural \\
\hline Watanabe (1960) & Japão & Natural \\
\hline
\end{tabular}




\begin{tabular}{|c|c|c|}
\hline Autor (es) & Procedência & Tipo de Infecção \\
\hline Basch (1966) & Malásia & Natural \\
\hline Freire (1967) & Brasil & Natural \\
\hline Nosaka et al. (1970) & Japão & Natural \\
\hline Campos et al. (1974) & Brasil & Natural \\
\hline Eduardo et al. (1976) & Filipinas & Natural \\
\hline Tang et al. (1977) & China & Natural \\
\hline Shien et al (1977) & China & Natural \\
\hline Moriyama et al. (1980) & Brasil & Natural \\
\hline Ribeiro et al. (1980) & Brasil & Natural \\
\hline Santos (1986) & Brasil & Natural \\
\hline \multicolumn{3}{|l|}{ Lebre } \\
\hline Tang (1950) & China & Natural \\
\hline \multicolumn{3}{|l|}{ Coelho } \\
\hline Chinone; Itagaki (1976) & Japão & Experimental \\
\hline Sakamoto et al. (1981) & Japão & Experimental \\
\hline \multicolumn{3}{|l|}{ Bubalino } \\
\hline Travassos (1944) & Brasil & Natural \\
\hline Tang (1950) & China & Natural \\
\hline Busetti et al. (1983) & Brasil & Natural \\
\hline \multicolumn{3}{|l|}{ Ovino } \\
\hline Janson (1889) & Japão & Natural \\
\hline Travassos (1944) & Brasil & Natural \\
\hline Tang (1950) & China & Natural \\
\hline Freire (1967) & Brasil & Natural \\
\hline \multicolumn{3}{|l|}{ Caprino } \\
\hline Travassos (1944) & Brasil & Natural \\
\hline Tang (1950) & China & Natural \\
\hline Basch (1966) & Malásia & Experimental \\
\hline Chinone; Itagaki (1976) & Japão & Experimental \\
\hline \multicolumn{3}{|l|}{ Camelo } \\
\hline Travassos (1944) & Brasil & Natural \\
\hline Tang (1950) & China & Natural \\
\hline \multicolumn{3}{|l|}{ Macaco } \\
\hline Tang (1950) & China & Natural \\
\hline \multicolumn{3}{|l|}{ Humano } \\
\hline Tang (1950) & China & Natural \\
\hline Chang; Li (1964) & China & Natural \\
\hline Asada et al. (1966) & Japão & Natural \\
\hline Ishii (1983) & Japão & Natural \\
\hline \multicolumn{3}{|l|}{ Suíno } \\
\hline Travassos (1944) & Brasil & Natural \\
\hline Tang (1950) & China & Natural \\
\hline \multicolumn{3}{|l|}{ Eurytrema dajii } \\
\hline \multicolumn{3}{|l|}{ Bovino } \\
\hline Bhalerao (1924) & Indonésia & Natural \\
\hline Handoko (1981) & Indonésia & Natural \\
\hline \multicolumn{3}{|l|}{ Eurytrema tonkinense } \\
\hline \multicolumn{3}{|l|}{ Bovino } \\
\hline Galliard; Ngu (1941) & Vietnã & Natural \\
\hline
\end{tabular}




\begin{tabular}{|c|c|c|}
\hline Autor (es) & Procedência & Tipo de Infecção \\
\hline \multicolumn{3}{|l|}{ Eurytrema satoi } \\
\hline Kobayashi (1920) & Japão & Natural \\
\hline \multicolumn{3}{|l|}{ Eurytrema rebele } \\
\hline Railliet (1924) & Indonésia & Natural \\
\hline \multicolumn{3}{|l|}{ Eurytrema parvum } \\
\hline Senoo (1907) & Japão & Natural \\
\hline \multicolumn{3}{|l|}{ Eurytrema ovis } \\
\hline Tubangui (1925) & Filipinas & Natural \\
\hline \multicolumn{3}{|l|}{ Eurytrema cladorchis } \\
\hline Tang; Tang (1977) & China & Natural \\
\hline \multicolumn{3}{|l|}{ Eurytrema escuderoi } \\
\hline \multicolumn{3}{|l|}{ Carabao } \\
\hline Eduardo et al. (1976) & Filipinas & Natural \\
\hline \multicolumn{3}{|l|}{ Eurytrema hydropotes } \\
\hline Tang; Tang (1975) & China & Natural \\
\hline \multicolumn{3}{|l|}{ Eurytrema médium } \\
\hline Tschertkova (1959) & Rússia & Natural \\
\hline
\end{tabular}

\section{Morfologia}

De acordo com Mattos Júnior e Vianna (1987) há muitos anos alguns problemas têm sido levantados quanto à diferenciação das espécies de Eurytrema spp. mais especificamente de E. coelomaticum e $E$. pancreaticum.

Autores como Looss e Cuffey (1907), Travassos (1944) e Eduardo, Manuel e Tongson (1976) dão ampla visão da tendência em classificar o Eurytrema spp. como espécies distintas. De acordo com os estudos realizados na China por Tang e Tang (1977), não só os aspectos morfológicos dos parasitos adultos são utilizados para esta distinção, mas a morfologia das formas larvares nos hospedeiros intermediários também é considerada. No Japão, Moriyama, Tsuji e Seto (1980) estudou as diferenças destas espécies através da cariotipagem e concluiu haver diferenças morfológicas e biométricas dos cromossomos, bem como as posições dos centrômeros destas duas espécies.

Conforme Travassos (1944) E. coelomaticum possui um corpo achatado com 10 a $13 \mathrm{~mm}$ por 6 a 7 $\mathrm{mm}$ de largura. Cutícula lisa, ventosa oral subterminal com 0,9 a $1 \mathrm{~mm}$ de diâmetro. Acetábulo no terço médio do corpo, com dimensões iguais às da ventosa oral. Faringe e esôfago delgados. Cecos intestinais 
delgados e sinuosos. Poro genital ligeiramente pósbifurcal mediano. Bolsa do cirro muito grande, geralmente claviforme. Testículos pós-acetabulares com zonas coincidentes e campos afastados. Ovários submedianos, pós-testiculares. Glândulas de Mehlis, espermateca e canal de Laurer presentes. Vitelinos laterais, extracecais e com alguns folículos intercecais. Ovos com $0,042-0,50 \mathrm{~mm}$ por 0,023 $0,03 \mathrm{~mm}$. Vesícula excretora simples e poro excretor terminal.

\section{Hospedeiros}

Os primeiros hospedeiros intermediários de $E$. coelomaticum podem ser espécies de caramujos terrestres do gênero Bradybaena. Após serem ingeridos, os ovos do trematódeo eclodem no tubo digestivo do molusco e lá iniciam o seu desenvolvimento. A espécie de caramujo terrestre, Bradybaena similaris, Férussac, (1821), foi descrita no Brasil como o primeiro hospedeiro intermediário de E. coelomaticum (RAGUSA; CAMPOS, 1976).

Após a saída do ovo, o miracídio penetra na parede do tubo digestivo do caramujo, onde se desenvolve em esporocisto-mãe ao produzir uma formação semelhante a uma massa arredondada, fixada à superfície externa da parede intestinal do molusco. Os esporocistos-mãe crescem, e no seu interior muitos esporocistos-filhos se desenvolvem. O desenvolvimento no interior do molusco completase em 90 dias após a infecção sob temperatura de $26^{\circ} \mathrm{C}$, enquanto em condições ambientais pode levar de 250 a 350 dias. Os esporocistos-filhos, quando se tornam alongados, abandonam o esporocisto-mãe e migram da superfície externa do trato digestivo à abertura respiratória, através da cavidade hemocelomática. Os esporocistos são eliminados pelo molusco através da abertura respiratória e chegam ao ambiente. Estes, por sua vez, contêm centenas de cercárias e sua morfologia é distinta para cada espécie de parasito. A emergência dos esporocistosfilhos normalmente ocorre horas antes do amanhecer (ITAGAKI; CHINONE, 1982).
Conforme Ragusa e Campos (1976), estudos realizados na região do Vale do Paraíba demonstraram que o molusco $B$. similaris é o primeiro hospedeiro intermediário de E. pancreaticum no Brasil.

De acordo com Yamaguti (1975), o segundo hospedeiro intermediário do E. coelomaticum e $E$. pancreaticum consiste em várias espécies de esperanças da família Tettigoniidae, gênero Conocephalus com várias espécies: C. maculatus, C. chinensis, C. melas, C. gladiatus, dentre outras. Itagaki e Chinone (1982) destacaram que o desenvolvimento da cercária em metacercária nesse hospedeiro intermediário é também influenciado pela temperatura, informando que o aumento da temperatura acelera o desenvolvimento, podendo atingir $\mathrm{o}$ amadurecimento em 15 dias, na temperatura de $30^{\circ} \mathrm{C}$.

A partir de um único ovo de Eurytrema são produzidos cerca de 100 esporocistos filhos, que por sua vez contém em torno de 200 cercárias, perfazendo um total de 20.000 formas infectantes, representadas pelas metacercárias. (ITAGAKI; CHINONE, 1982).

Tang e Tang (1977) relataram que as metacercárias são encontradas na cavidade abdominal do inseto, podendo ou não atingir a região do tórax. Seu número pode variar de 1 a 461 metacercárias por inseto.

Segundo Basch (1966), os hospedeiros definitivos adquirem a infecção ao ingerir as esperanças infectadas juntas com as pastagens. Estudos realizados por Chinone e Itagaki (1976), Sakamoto et al. (1980) e Chinone; Fukase e Itagaki (1984) demonstraram que as metacercárias são liberadas dos cistos no duodeno e migram para os ductos pancreáticos. O período pré-patente é de 90 a 100 dias e o período patente é de um a dois anos. Vários animais foram utilizados para as infecções experimentais, mas somente os caprinos, coelhos, camundongos $B A L B / c$ nude e gatos demonstraram sensibilidade, sendo os caprinos e os camundongos $B A L B / c$ nude foram os mais susceptíveis às infecções. 
Mattos Júnior e Vianna (1987) estudaram o ciclo biológico do $E$. coelomaticum infectando experimentalmente exemplares de $B$. similaris, com ovos de E. coelomaticum, e as larvas desenvolvidas nesse hospedeiro foram estudadas morfologicamente, notando-se que a morfologia dos esporocistos expelidos pode caracterizar a espécie E. coelomaticum. O número de esporocistos expelidos por caramujo variou muito. Ortópteros das famílias Gryllidae, Acridiidae e Tettigoniidae foram capturados nos municípios de Lorena e Pindamonhangaba estado de São Paulo, e examinados à procura de metacercárias de $E$. coelomaticum. Apenas duas espécies de "esperanças", os tettigoniídeos Conocephalus saltator e Conocephalus sp. albergavam metacercárias de E. coelomaticum. Infecção experimental destes insetos, com esporocistos expelidos de $B$. similaris infectados experimentalmente, comprovou serem estes tettigoniídeos o segundo hospedeiro intermediário de E. coelomaticum, o qual foi identificado pela primeira vez, no Brasil. Infecções experimentais feitas em camundongos albinos linhagem BALB/c normal, em ratos linhagem Wistar e em cabras, utilizando-se "esperanças" infectadas experimentalmente e naturalmente, bem como com metacercárias dissecadas destes insetos, apresentaram resultados positivos apenas nas cabras. Os exemplares de $E$. coelomaticum coletados das cabras experimentalmente infectadas eram bem menores do que os normalmente coletados em bovinos. A prevalência média de E. coelomaticum em $B$. similaris, nos municípios estudados variou entre $9,7 \%$ e 20,1\%, enquanto que em Conocephalus spp. variou entre $0,6 \%$ e $70 \%$. As alterações histopatológicas encontradas no molusco hospedeiro intermediário foram causadas pela compressão das larvas que atingiam principalmente a glândula digestiva e a ovotestis (MATTOS JÚNIOR; VIANNA, 1987).

\section{Distribuição Geográfica}

A euritrematose possui ampla distribuição geográfica. Segundo Manninger e Mocsy (1973) foi registrada em bovinos e caprinos na Índia, Indonésia, Japão, América do Sul, e na Ásia Central o registro se deu em camelos.

Vários autores a registraram em outros animais, como em búfalos, além de bovinos, ovinos e caprinos no Japão, Indochina, Índia, e América do Sul, (LAPAGE, 1956; NIEBERLE; COHRS, 1970).

O E. pancreaticum foi observado no Brasil entre 1970 e 1979 por vários pesquisadores, entre eles destacam-se Brito, Francis e Carvalho (1981) que identificaram sua ocorrência nos Estados de Minas Gerais, Rio de Janeiro, Santa Catarina, Rio Grande do Sul e São Paulo.

Horta (1918) diagnosticou no Brasil o primeiro caso de infecção por Eurytrema spp. em ruminantes, e o que mais chamou sua atenção foi a intensidade de infecção, mesmo em bovinos jovens. As lesões produzidas por esse trematódeo em pâncreas de bovinos foram posteriormente descritas por: Figueiredo (1928), Torres e Pinto (1936), Ribeiro et al. (1980) e Correa et al. (1984).

O estudo mais detalhado descrito sobre a prevalência da euritrematose foi realizado por Brant (1962), que observou 1.876 animais em treze municípios do estado de Minas Gerais. Neste estudo verificou-se que a intensidade do parasitismo variou muito pouco em zonas de criação, mas na zona metalúrgica, três municípios observaram prevalências superiores a 30\%: Itabira $(33,8 \%)$, Bom Jesus do Amparo (49,1\%) e Ferros (67,5\%).

Costa et al. (1986) relataram a ocorrência de $E$. coelomaticum parasitando canais pancreáticos de bovinos nos Estados do Mato Grosso do Sul, Minas Gerais, Paraná, Rio Grande do Sul, Rio de Janeiro, Santa Catarina e São Paulo, em ovinos do Rio Grande do Sul, em caprinos de Minas Gerais e Rio de Janeiro, e a ocorrência de E. pancreaticum em pâncreas de bubalinos no Estado do Paraná. 
Mattos Júnior e Vianna (1987) relatam também que levantamentos efetuados através de necropsias, visitas a matadouros e exame de fezes, demonstraram que mais de $80 \%$ das propriedades existentes no município de Pindamonhangaba possuem animais parasitados pelo referido trematódeo. Além de Pindamonhangaba, outros municípios da região do Vale do Paraíba (Lorena, Guaratinguetá, Roseira, Aparecida do Norte e Taubaté) apresentaram bovinos acometidos pela doença.

Santos e Prata (1998), objetivando verificar a presença de infecção por Eurytrema spp. acompanharam o abate de 5.301 bovinos, procedentes dos estados de São Paulo, Minas Gerais, Goiás e Mato Grosso. Desses verificou-se a presença da parasitose em 1.155 animais, correspondendo a $22,9 \%$.

Azevedo et al. (2000), em um estudo retrospectivo em bovinos abatidos sob o regime de Inspeção Federal no norte do Estado do Paraná, relataram a prevalência e a distribuição geográfica da euritrematose em bovinos. A prevalência média identificada foi de $12,1 \%$, variando de $8,3 \%$ na região de São João do Caiuá, a 40,5\% na região de Ponta Grossa. O resultado do estudo aponta que a euritrematose prevalece dentro do Estado devido a fatores biológicos do trematódeo, dos hospedeiros intermediários, das condições ambientais e de certas particularidades de cada região.

Ainda no Paraná, Yamamura (2005) reportou pelos dados de registros realizados pelo Serviço de Inspeção Federal, durante o período de 1976 a 1980, em um matadouro frigorífico, localizado em Londrina, no total de 450.614 bovinos procedentes de quatro estados da federação, 94.991 pâncreas de bovinos estavam parasitados com E. coelomaticum, e em todos os estados apresentaram animais infectados com este trematódeo. O Paraná com 221.095 $(49,06 \%)$ bovinos abatidos neste período, procedentes de 48 municípios, totalizou 82.355 (37,24\%) bovinos com pâncreas parasitados, com variação de taxa média de positividade de 33,2\% em 1976 a 40,9\% em 1980. Umuarama foi o município com maior população bovina abatida no estado, 16.750 e apresentou 34\% de animais parasitados. Londrina, com $9,5 \%$ de população bovina abatida no Paraná, teve o índice médio de $63 \%$ de animais positivos. Um dos índices mais altos foi constatado no município de Maria Helena, com $80 \%$ de positividade em uma população de $2,45 \%$ de bovinos abatidos no estado. O estado de Mato Grosso do Sul com 177.772 (39,45\%) bovinos procedentes de 29 municípios, 9.742 $(5,48 \%)$ tiveram pâncreas infectados com Eurytrema. O índice médio de positividade para o estado foi de 5,4\%, com variação mínima de 2,1\% em 1976 a 12,4\% em 1980. Este índice apresentou crescente de 1976 a 1980, provalvelmente devido ao trânsito de animais entre este estado e o Paraná, e ainda associada a presença dos hospedeiros intermediários. O município de Amambaí, com o maior volume de abate (42.874), apresentou 2.406 animais infectados e o maior volume de animais infectados foi o município de Iguatemi com 2.471, representando 7,6\% de infectados de 32.434 animais abatidos. O estado de São Paulo, com 47.173 bovinos abatidos, teve 2.738 infectados e com índice de positividade de 5,8\% e com uma variação de 3,0\% em 1979 a 11,3\% em 1977. O município de maior volume de abate foi Pereira Barreto com 7.102 bovinos e com 170 animais infectados e aquele município de maior volume de animais infectados foi Teodoro Sampaio com 353 pâncreas parasitados de 7.008 animais abatidos. Os estados de Mato Grosso e Goiás tiveram 3.841 e 733 animais abatidos respectivamente e com $108(2,8 \%)$ e 48 (6,5\%) pâncreas parasitados. No Mato Grosso, o município de Chapada dos Guimarães foi o que apresentou maior índice de positividade com 56 (19,3\%) animais infectados de 290 bovinos examinados e em Goiás foi o município de Anápolis, com 136 (33,8\%) animais examinados 46 estavam parasitados. Também foram abatidos 1.384 bubalinos neste estabelecimento no mesmo período e $52(3,7 \%)$ animais apresentaram pâncreas infectados. A maior porcentagem de positividade verificada foi no Paraná, com $8,0 \%$, 
seguida de Mato Grosso, com 7,5\% e em São Paulo e Mato Grosso do Sul com 1,0\% e 0,9\% respectivamente. A maioria destes bubalinos parasitados era procedente de locais onde constatou também a ocorrência de parasitismo em bovinos.

Bassani (2005) acompanhou o abate de 1828 bovinos em um frigorífico localizado no município de Campo Mourão - PR, no período de Junho de 2003 a Maio de 2004, e observou que 47,8\% (874/1828), dos pâncreas estavam parasitados pelo E. coelomaticum.

\section{Patogenia e Patologia}

Em relação à patogênese, Figueiredo (1928) descreveu as lesões produzidas pelo $E$. coelomaticum. Este autor descreveu um processo de pancreatite intersticial crônica com a proliferação de tecido conjuntivo, acarretando a destruição dos ácinos glandulares com conservação das ilhotas de Langerhans e infiltrações linfocitárias, sendo este aspecto também assinalado ao redor dos pontos onde são encontrados os parasitos, assim como a reação em torno dos ovos que se achavam distribuídos no parênquima pancreático. Pinto e Almeida (1935) confirmaram estas lesões, sendo que mais tarde Torres e Pinto (1936) estudaram o assunto mais detalhadamente, observando lesões tanto microscópicas quanto macroscópicas.

a) Patologia causada pelas formas adultas de $E$. coelomaticum

Torres e Pinto (1936) descreveram a ação do parasitismo de formas adultas de E.coelomaticum em pâncreas de bovino naturalmente infectado e caracterizaram as lesões macroscópicas e microscópicas bem como a implicação que os ovos exercem neste mecanismo. Trabalhos como de Kono e Fukuyoshi (1966); Nosaka, Ashizawa e Nagata (1970) também investigaram o mecanismo da ação patológica dos ovos na infecção de Eurytrema spp.

Basch (1966) estudou a biologia de E. pancreaticum, também fez observações sobre a patologia e registrou alterações microscópicas e macroscópicas e ainda fez correlações entre o tamanho e a coloração dos parasitos e as condições dos órgãos parasitados.

Campos et al. (1974) verificaram que havia correlação positiva e significativa entre o número de parasitos e o peso de pâncreas parasitado com $E$. pancreaticum, ou seja, à medida que aumenta o número de parasitos aumenta também o peso do pâncreas.

Headley (2000) observou em investigações macroscópicas realizadas na Unidade de Patologia Veterinária da Universidade Federal de Santa Maria, que não somente os ductos contendo os parasitas são atingidos. Ocasionalmente todo tecido acinar do trato pancreático também é atingido, sendo substituído por tecido fibroso. Proliferação do tecido conectivo induz a destruição dos ácinos glandulares, conservando as ilhotas de Langerhans. O infiltrado linfático, tem sido evidenciado ao redor dos pontos onde os trematódeos são encontrados.

Oliveira e Bechara (1988) avaliaram os aspectos microscópicos do pâncreas do bovino parasitado por E.coelomaticum e evidenciaram um quadro de pancreatite fibrosante crônica, caracterizado por exuberante fibroplasia que substituía extensas áreas do parênquima pancreático e por copiosos infiltrados celulares inflamatórios do tipo mononuclear, distribuídos de forma difusa ou em focos pelo tecido glandular, ora entre os ácinos, ora ao redor dos ductos pancreáticos. Foram observados ainda, degeneração pancreática; calcificação distrófica e ossificação membranosa metaplásica; atrofia de ácinos pancreáticos por compressão fibrosa e presença de ovos de Eurytrema spp. houve também comprometimento do pâncreas endócrino com a redução de números e dimensões das ilhotas de Langerhans.

Belém, Oliveira e Padovani (1994) caracterizaram as lesões pancreáticas de acordo com suas características histológicas e extensão desde o epitélio dos ductos até o parênquima, em pelo menos 50\% dos cortes histológicos examinados, e podem ser enquadradas em quatro graus distintos: 
- Grau I: lesões ausentes ou incipientes e geralmente restrita ao epitélio dos ductos;

- Grau II: espessamento moderado, porém nítido, das paredes dos ductos associado à hiperplasia epitelial, infiltrado inflamatório e/ou fibroplasia discreta sem, contudo, ocorrer tendência à diminuição da luz;

- Grau III: alterações confinadas aos ductos interlobulares, principalmente de maior calibre, e traduzidas, sobretudo, por espessamento marcante das paredes devido à fibroplasia e reação inflamatória que, muitas vezes, ocasionam estenose;

- Grau IV: ocorrência de lesões ductais descritas para o grau III associadas a outras parenquimatosas de extensão variável, caracterizadas principalmente por reação inflamatória e fibrosa.

Em cortes histológicos de pâncreas parasitados com E. pancreaticum Correa et al. (1984) observaram granulomas parasitários periductais com parasitas e ovos eventualmente invadindo o parênquima, somente em lóbulos adjacentes a ductos interlobulares com mais de um $\mathrm{mm}$ de diâmetro. $\mathrm{O}$ tecido conjuntivo, ao redor dos ductos parasitados inter e intralobulares, mostravam forte hiperplasia de fibras do colágeno.

As principais alterações macroscópicas do pâncreas foram relatadas por Graydon et al. (1992), sendo: alteração do volume em muitas vezes, com seu comprimento extremamente diminuído, coloração pálida, consistência dura.

Yamamura (1989) classificou as lesões macroscópicas produzidas por E. coelomaticum em três tipos:

- Tipo I: ausência de lesões macroscópicas nos ductos e parênquima;

- Tipo II: presença de lesões macroscópicas somente nos ductos;

- Tipo III: presença de lesões macroscópicas nos ductos e parênquimas.
Os resultados mostraram que o parasito causa alterações nas células acinares e nos casos mais extremos havia a ausência destas células, decorrente da destruição acarretada pela fibrose.

Sakamoto et al. (1980), notaram que não havia diferença significativa no tamanho dos parasitos obtidos de infecção experimental de caprino e de camundongo nude. À medida que aumentou a carga parasitária, também houve atrofia dos órgãos e os parasitos obtidos em infecção experimental nestes animais tiveram tamanho maior, se comparados com aqueles colhidos de bovinos naturalmente infectados. Ressaltaram ainda os autores que a troca regressiva dos tecidos acinares foi vista em pâncreas de camundongos infectados. Por este achado, eles consideram que o parasito teve severa patogenicidade sobre o tecido acinar de pâncreas.

Mattos Júnior e Vianna (1987) observaram que os espécimes de E. coelomaticum colhidos de caprinos experimentalmente infectados eram de tamanho menor do que os colhidos dos bovinos naturalmente infectados.

b) Patologias Causadas por Agentes Bacterianos Secundários

Quando se estuda a patogenicidade de um agente parasitário, a atenção é sempre dirigida ao agente primário. No entanto, a severidade do quadro pode ser acarretada por agentes considerados secundários, notadamente quando o parasito cria um estado imunodepressor, como mostraram os trabalhos desenvolvidos por Aitken et al (1979) com animais infectados com $F$. hepatica.

Euzeby (1954) assinalou que a infecção intercorrente pode ser realizada pela transferência de agentes bacterianos pela migração dos trematódeos, como na fasciolose. Entre estes se incluem agentes anaeróbicos como Clostridium spp. assim facilitando o aparecimento de hepatite necrótica e aumentando o potencial patogênico dos esporos de Clostridium novyi, evidenciado pelo trabalho realizado por Bagadi e Sewell (1973). 
No entanto, a maioria dos trabalhos cita a ocorrência de Escherichia coli, como um dos agentes enterobacterianos que aparecem com maior freqüência e porcentagem, como pode ser visto no trabalho de Ogunrinade e Adegoke (1982), que trabalharam com infecção de F. hepatica. No Brasil, também Yamamura, Horner e Lopes (1995) relataram em seus estudos que a bactéria mais freqüente encontrada foi $E$. coli. No pâncreas não existiu evidência da invasão de formas patogênicas de $E$. coli como septicêmica, entérica ou enterotoxêmica que se manifestam principalmente em bezerros, citadas por Gay (1965) e Gay e McKay (1964). Possivelmente a $E$. coli adere-se à mucosa e coloniza novas superfícies celulares do epitélio pancreático provocando reações celulares, podendo levar à formação de granulomas como citou Guerreiro et al. (1984).

A necessidade de averiguar a real extensão da patologia da euritrematíase bovina se deve a problema de saúde pública, uma vez que este órgão sofre um processo de industrialização na extração de hormônios, entre eles a insulina, que é destinada para o uso humano.

\section{Diagnósticos}

A insuficiência de referências bibliográficas relativas ao diagnóstico de euritrematose foi assinalada por Côdo (1952), observando que embora houvesse elevada infecção, que esta só era detectada em matadouros, após o exame do pâncreas.

As técnicas coproparasitológicas para a pesquisa de ovos de trematódeos encontradas na literatura dão especial referência a $F$. hepatica, Schistosoma mansoni e Paramphistomum spp.

Ragusa e Campos (1976) realizaram pelo método de Ueno e Watanabe (1968), exame de fezes de 1.171 bovinos, demonstrando que 584 animais eram disseminadores de ovos de Eurytrema spp.

Chinone e Itagaki (1976) descreveram um método coproparasitológico para a contagem de ovos do parasito nas fezes. Correa et al. (1984) trabalharam com provas intradérmicas, utilizando uma suspensão de parasitos após terem sido adequadamente preparados em diversas diluições. A diluição do antígeno para 1: 500 foi considerada ideal, fazendose a leitura meia hora após a inoculação. $\mathrm{O}$ erro médio das provas falso-negativas foi em torno de 29\% (resultados negativos em animais doentes).

Vianna (1984) padronizou a técnica coproscópica de sedimentação para a concentração de ovos de Eurytrema spp.

Mattos Júnior e Vianna (1987), destacam que freqüentemente, em infecções por helmintos de ruminantes ou de outras categorias de hospedeiros, o número de ovos eliminados com as fezes não está necessariamente relacionado com o número de vermes albergados, mesmo em se tratando de espécies que eliminam suas formas evolutivas na luz intestinal.

No caso dos parasitos que não depositam diretamente suas formas de eliminação no tubo digestivo, é sabido que modificações do tecido onde se localiza o parasita (fibrose, por exemplo), podem retardar ou obstruir a passagem dessas formas. Portanto, considerado isoladamente ou ligado a outros, esse fenômeno teria, possivelmente, um papel importante no número de ovos eliminados por parasitos do gênero Eurytrema spp.

Belém, Oliveira e Padovani (1992) relatou que os seguintes aspectos, ligados ao diagnóstico da infecção por Eurytrema sp. 1) aferição da prova do filme como método de triagem; 2) estudo da distribuição de probabilidades do número de parasitas por animal e do OPG, este determinado segundo a técnica de Dennis, Stone e Swanson (1954) com modificações; 3) estimativa da carga parasitária em função da contagem de ovos por grama de fezes (OPG); 4) graduação das lesões microscópicas de pâncreas e sua associação com a carga parasitária e o OPG; 5) avaliação da intradermo-reação cervical (IDR). Todas estas investigações, à exceção da IDR, foram conduzidas em bovinos adultos, oriundos de 
municípios da mesorregião do sudoeste Paulista e abatidos em São Manuel ou necropsiados na Faculdade e Medicina Veterinária e Zootecnia Botucatu (FMVZ). As seguintes conclusões foram obtidas: $1^{\text {a }}$ ) a prova do filme deve ser desconsiderada para triagem dos casos clínicos; $2^{\mathrm{a}}$ ) a intensidade média de parasitismo é baixa na região em estudo e a prevalência situa-se entre $7,2 \%$ e $9,8 \% ; 3^{\mathrm{a}}$ ) um único exame coproparasitológico, por meio da técnica referida, tem $94,2 \%$ de probabilidade de diagnosticar casos de infecção pelo Eurytrema spp. independentemente da carga parasitária do hospedeiro; $4^{\mathrm{a}}$ as lesões microscópicas de pâncreas foram classificadas em quatro diferentes graus, segundo a extensão desde a mucosa dos ductos pancreáticos, mas, em nenhum caso estudado, podese consignar qualquer tipo de alteração mais grave reportada na literatura; $5^{\mathrm{a}}$ ) não se consegue uma boa estimativa da carga parasitária de um bovino, nem do grau de lesão correspondente, quando se toma o OPG como parâmetro; $6^{\mathrm{a}}$ ) pode-se verificar que o número de parasitas por animal se presta melhor que o OPG para caracterizar graus de lesão. Quanto à IDR, pesquisa efetuada em bovinos adultos abatidos no matadouro de Viçosa e procedentes de municípios da microrregião, concluiu-se que: $1^{\circ}$ ) podem ser consideradas positivas as IDR que apresentem aumento relativo das pápulas superior a $2,5 \mathrm{~mm}$ e negativas aquelas iguais ou inferiores; $2^{\circ}$ ) o antígeno de Eurytrema spp. que consistiu de um extrato salino bruto de parasitas adultos, deverá conter, no momento de sua utilização, concentração de proteína equivalente a $250 \mathrm{mg} / \mathrm{mL}$.

\section{Controle Terapêutico}

a) Teste in vivo

Muitas drogas foram testadas, em função da atividade anti-helmíntica, principalmente contra os trematódeos de ruminantes. Kukharenko (1971) testou drogas como hexacloroparaxilol, hexide, hexacloroetano, cyazide, sulfeno e clorofos e nenhuma destas drogas foi capaz de agir contra o
Eurytrema spp. em ruminantes. O mesmo resultado foi obtido por Nosaka, Ashizawa e Nagata (1970), quando empregaram o bithionol, bithionol sulfóxido, thiabendazole e piperazina.

Sakamoto et al. (1980) quando testaram nitroxinil e praziquantel, notaram evidências de serem efetivos anti-helmínticos para E. coelomaticum, baseandose nos dados relativos a decréscimo da quantidade de ovos eliminados pelas fezes, bem como a degeneração destes, reforçando, com o encontro de trematódeos degenerados. O encontro de células em regeneração ativa dos tecidos tissulares, observados em exames histopatológicos de pâncreas de animais tratados, reforça ainda mais as atividades destas drogas. Estes mesmos autores sugerem que o praziquantel seja um anti-helmíntico ideal contra trematódeos do gênero Eurytrema spp. e assim observaram que, utilizando a droga, havia um aumento de ovos anormais de E. coelomaticum.

Os testes anti-helmínticos em animais infectados experimentalmente foram realizados por Kono et al. (1980) seguindo as indicações de Sakamoto et al (1980) que utilizaram o praziquantel em caprinos e que mostrou ser efetivo, observados pelo decréscimo da quantidade de ovos nas fezes, e o encontro de pequenas quantidades de parasitos e da observação de que no órgão haviam muitos leucócitos. Assim demonstraram uma eliminação gradual do parasito e a recuperação do tecido, com a proliferação do epitélio do ducto pancreático. Neste mesmo experimento os autores testaram triclabendazole e este não mostrou nenhuma atividade anti-helmíntica para trematódeo pancreático.

Araújo e Belém (1993) concluíram que o albendazole, mesmo em altas dosagens $(17,5 \mathrm{mg} / \mathrm{kg}$ de peso vivo), não alterou de forma significativa a contagem de ovos por grama de fezes (OPG) de Eurytrema spp.

$\mathrm{Na}$ literatura sobre anti-helmínticos utilizados no controle da F. hepatica, como de Armour e Bogan (1982) notou-se que havia uma seletividade muito grande das drogas testadas que agem sobre este 
parasito e sendo mais restrito quando se tratou de fase parasitária abaixo de quatro semanas após infecção. Entre estas drogas incluíam-se dianfenetide. Mas esta não foi indicada para bovino, e triclabendazole, que não mostrou ser ativo para trematódeo de localização pancreática.

\section{b) Teste in vitro}

Foram poucos os trabalhos que tentaram observar a atuação de medicamentos anti-helmínticos sobre formas adultas de trematódeos de localização pancreática.

Sakamoto et al. (1984) desenvolveram testes in vitro, utilizando cercárias de E. coelomaticum, obtidos de esporocistos de B. similaris. A manutenção destas cercárias era feita em meios básicos sintéticos, contendo $20 \%$ de soro fetal bovino, $0,812 \%$ de lactoalbumina hidrolisável, $0,625 \%$ de extrato seco e $0,5 \%$ de glicose, incubados a $28^{\circ} \mathrm{C}$ e acondicionados em tubos de Falcon, contendo $5 \mathrm{ml}$ do meio. Foram testadas 88 drogas que eram dissolvidas ou suspensas em propileno glicol na concentração de 0,002 , 0,02, 0,2, 2,0 e 20,0 miligramas por mililitro. As alterações morfológicas das cercárias foram observadas por 24 horas, através do uso de microscópio com contraste de fase e também foi testada a motilidade. As sobrevivências das cercarias foram menores entre as drogas como praziquantel, derivados de quinolina-cobre 8, quinolina, 3,5,5', tribromo salicyl-0-toluidina, 3,5-dibromo-3'trifluorometil salicilanilida, e 2,5-dicloro-4'nitrosalicilanilida-derivado de salicilanilida, um derivado de benzimidazole-mebendazole e derivado de fenol-nitroxinil.

Yamamura (1989), utilizou exemplares de $E$. coelomaticum que foram submetidos a estudos experimentais de sobrevivência em meios de cultivos, como Gay ABC, 199 e soro de ovino e neste último meio, o trematódeo sobreviveu por um período superior a 1.000 horas. O soro de ovino também serviu como meio de escolha para os testes de drogas antihelmínticos in vitro. As drogas testadas: nitroxynil, rafoxadine, tricabendazole e closantel, tanto nos testes in vitro como in vivo, não foram capazes de determinar a morte deste trematódeo.

\section{Considerações finais}

Sabe-se que foram mais estudadas a morfologia, prevalência em algumas regiões e as alterações anátomo-patológicas, mas outros aspectos continuam a merecer novos estudos, uma vez que a freqüência do E. coelomaticum em bovinos nacionais é bem conhecida.

No Brasil, os estudos sobre esta parasitose se iniciaram no Rio Grande do Sul em 1961, contudo, voltavam-se unicamente para os principais nematóides gastrointestinais de ruminantes. Somente a partir de 1976 é que se tomou conhecimento de estudos biológicos do E. coelomaticum, no entanto poucos trabalhos foram desenvolvidos desde então.

Neste contexto, é necessário salientar que a euritrematose apresenta-se geralmente na forma subclínica e os produtos anti-helmínticos disponíveis atualmente tem se mostrado ineficiente para combater a euritrematose, então torna-se de grande importância maiores estudos voltados a epidemiologia, sintomas no hospedeiro definitivo, práticas racionais para o controle dos hospedeiros intermediários, com o propósito de facilitar as medidas de controle que envolvem esta parasitose.

\section{Referências}

AITKEN, M. M.; HUGES, D. L.; JONES, P. W.; HALL, S. A.; SMITH, G. S. Immunological responses and flukes infected and fluke free cattle to Salmonella dublin and other antigens. Research in Veterinary Science, London, v.27, n.3, p.306-312, 1979.

ARAÚJO, J. V.; BELÉM P. A. D. Efeito anti-helmíntico do albendazole sobre a contagem de ovos de Eurytrema sp (Trematoda) em fezes de bovinos. Arquivo Brasileiro de Medicina Veterinaria e Zootecnia, Belo Horizonte, v.45, n.1, p.111-114, 1993.

ARMOUR, J.; BOGAN, J. A. Anthelmintics for ruminants. British Veterinary Journal, London, v.138, n.5, p.371-382, 1982. 
AZEVEDO, J. R.; MANNIGEL, R. C.; BORBA,T. R.; BARBIÉRI, A. W.; OLIVEIRA, D. C. L.; HEADLEY,S. A.; JANEIRO, V. Prevalence and geographical distribution of bovine eurytrematosis in cattle slaughtered in Northern Paraná, Brazil. Maringá: CESUMAR, 2000.

BADAGI, H. O.; SEWEL, M. M. H. Experimental studies on infections necrotic hepatitis (black disease) of sheep. Research in Veterinary Science, London, v.15, p.53-61, 1973.

BASCH, P. F. Patterns of transmission of trematode Eurytrema pancreaticum in Malaysia. American Journal of Veterinary Research, Chicago, v.27, n.116, p.234-240, 1966.

BASSANI, A. C. Estudo epidemiológico e patológico da euritrematose bovina. 2005. Tese (Mestrado em Ciência Animal - Concentração Sanidade Animal) - Universidade Estadual de Londrina, Londrina.

BELÉM, P. A. D.; OLIVEIRA, M. R.; PADOVANI, C. R. Alterações pancreáticas em bovinos naturalmente infectados por Eurytrema sp e sua associação com a carga parasitária e o número de ovos por grama de fezes (OPG). Brazilian Journal of Veterinary Research and Animal Science, São Paulo, v.31, n.3-4, p.273-281, 1994.

BELÉM, P. A. D; OLIVEIRA, M. R; PADOVANI, C. R. Adaptação da técnica de Denis, Stone \& Swanson para diagnóstico copro-parasitológico de infecção natural por Eurytrema sp em bovinos. Brazilian Journal of Veterinary Research and Animal Science, São Paulo, v.29, n.2, p.303-307, 1992.

BELÉM, P. A. D.; SILVA, J. C. P.; VIEIRA, D. Diabete mellitus em bovino. In: ENCONTRO DE PESQUISAS VETERINÁRIAS, 11., Jaboticabal, 1986. Resumo... Jaboticabal, 1986.

BORAY, S. C. Flukes of domestic animals. In: GAAFAR, S. M.; HOWARD, W. E.; MARSH, R. E. Parasites pests and predators (Word Animal Sciences, $B_{2}$ ). Glenfield: Elsevier Sci. Publis, 1985.

BRANT, P. C. Freqüência de algumas parasitoses em carcaças e vísceras de bovinos abatidos em Belo Horizonte. Arquivo Brasileiro de Medicina Veterinaria e Zootecnia, Belo Horizonte, v.14, p.127-132, 1962.

BRITO, D. B.; FRANCIS, M.; CARVALHO, E. C. R. Patogenicidade do Eurytrema coelomaticum (Giard \& Billet, 1892, LOOSS, 1907). Revista Brasileira de Medicina Veterinária, Rio de Janeiro, v.4, n.4, p.17, out./dez. 1981.

BURGgRAAf, H. Pâncreas-distomatose. Tijdsch diergeneesk, Utricht, v.62, n.8, p.469-481, 1935.

BUSETTI, E. T.; PASKE, A.; RUIS, M. C. E.; TOMAZ, V.; GOLINELLI, A. Helmintos parasitos de Bubalus bubalis no Estado do Paraná. Arquivo Brasileiro de Medicina Veterinaria e Zootecnia, Belo Horizonte, v.35, n.3, p.399404, 1983.
CAMPOS, M. S.; RAGUSA, A. L.; MIGUEL, O.; ISHIZUKA, M. M. Correlação entre o número de parasitas e peso de pâncreas em bovinos naturalmente infestados por Eurytrema pancreaticum, Janson 1889 (Trematoda, Dicrocoeliidae); diferença de peso entre pâncreas parasitados e não parasitados. Revista da Faculdade de Medicina Veterinária e Zootecnia da Universidade de São Paulo, São Paulo, v.11, p.295-299, 1974.

CHINONE, S.; FUKASE, T.; ITAGAKI, H. Experimental infection of domestic cats with Eurytrema coelomaticum (Trematoda: Dicrocoeliidae). Japanese Journal of Parasitology, Tokyo, v.33, n.1, p.29-39, 1984.

CHINONE, S.; ITAGAKI, H. Development of Eurytrema pancreaticum (trematoda). II - Development in definitive host. Bulletin of the Azabu Veterinary College, Sagamihara, v.1, n.2, p.73-81, 1976.

CODO, V. Teste cutâneo para diagnóstico da euritrematose em bovinos. Revista Ceres, Viçosa, v.9, n.50, p.132-138, 1952.

CORREA, O. Doenças parasitárias dos animais domésticos. 4. ed. Porto Alegre: sulina, 1983.

CORREA, W. M.; CORREA, C. N. M.; FERREIRA, A. C.; PAES, A. C. Eurytrema pancreaticum: clínica e diagnóstico em bovinos. A Hora Veterinária, Porto Alegre, v.4, n.19, p.31-34, 1984.

COSTA, H. M. A; LEITE, A. C. R.; GUIMARÃES, M. P.; LIMA, W. S. Distribuição de helmintos parasitas de animais domésticos no Brasil. Arquivo Brasileiro de Medicina Veterinaria e Zootecnia, Belo Horizonte, v.38, n.4, p.465$579,1986$.

DENIS, W. R.; STONE, W. M.; SWANSON, L. E. A new laboratory and field diagnostic test for fluke ova in feces. Journal of the American Veterinary Medical Association, Schaumburg, v.124, n.922, p.47-50, 1954.

EDUARDO, S. L.; MANUEL, M. F.; TONGSON, M. S. Eurytrema escuderoi, a new species and two other previously knows species of the genous Eurytrema. Philippine Journal of Science, Manila, v.15, n.1-2, p.104116, 1976.

EUZEBY, J. Parasitisme et infection. Cahiers de Medicine Veterinaire, Paris, n.2, 1954.

FIGUEIREDO, C. B. Sobre as lesões do pâncreas de boi produzidos pelo Eurytrema pancreaticum. Rio de Janeiro: Memorial Instituto Oswaldo Cruz, 1928. Supl.

FOX, N. J.; MOSLEY, J. G.; VOGLER, G. A.; AUSTIN, J. L.; REBER, H. A. Pancreatic function in domestic cats with pancreatic fluke infections. Journal of the American Veterinary Medical Association, Schaumburg, v.178, n.1, p.58-60, 1981. 
FREIRE, J. J. Fauna parasitária riograndense. I Introdução, boi, ovelha e cabra. Revista de Medicina Veterinária, São Paulo, v.3, n.1, p.40-55, 1967.

FREITAS, M. G. Helmintologia veterinária. Belo Horizonte: Rabelo \& Brasil, 1977.

GASTE, L. Avaliações laboratoriais em bovinos infectados e não infectados pelo Eurytrema coelomaticum. 1991. Tese (Doutorado em Medicina Veterinária- Área: Clínica)- Faculdade de Medicina Veterinária e Zootecnia da Universidade Estadual Paulista "Julio de Mesquita Filho", Botucatu.

GAY, C. C. Escherichia coli and neonatal disease of claves. Bacteriological Reviews, Baltimore, v.29, n.1, p.75-101, 1965.

GAY, C. C.; MCKAY, K. A. Studies on colibacilosis of claves. II. A clinical evolution of the efficiency of vaccination of the dane as a means of preventing colibacilosis of the calf. Canadian Veterinary Journal, Ottawa, v.5, p.297-308, 1964.

GIARD, A.; BILLET, A. Sur quelques trematodes parasites dês boeufs du Tonkin. C.R. Social Biology, Madison, v.4, p.613-615, 1892.

GRAYDON, R. J.; CARMICHAEL, I. H.; SANCHES, M. D.; WEIDOSARI, E.; WIDJAYANTI, S. Mortalities and wasting in Indonesian sheep associated with the trematoda Eurytrema pancreaticum. Veterinary Record, London, v.131, n.19, p.443, 1992.

GUERREIRO, M. G.; OLIVEIRA, S. J.; SARAIVA, D.; WIEST, J. M.; LIEBRKNECHT, F.; POESTER, F.; DIAS, J. C. A., FERNANDES, J. C. T.; LANGELO, H. A.; BAPTISTA, P. J. H. P. Bacteriologia especial. Porto Alegre: Sulina, 1984.

HARADA, H.; WATO, M.; FUJIWARA, N. Eurytrema infection in dairy cattle. Journal of Veterinary Medicine, Berlin, v.707, p.328-331, 1980.

HARRIS, D. G. Eurytrema pancreaticum. Journal of the Royal Army Veterinary Corps, Aldershot, v.23, n.1, p.2324, 1952.

HEADLEY, S. A.; Bovine eurytrematosis: life cycle, pathologic manifestations and public health considerations.. CESUMAR, Maringá, v.2, n.2, p.59-62, 2000.

HORTA, P. P. Distomatose pancreática e glicosúria em bovinos. A lavoura, Rio de Janeiro, v.22, n.3-4, p.157-158, 1918.

ITAGAKI, H; CHINONE, S. Histories of rumen flukes and pancreas of ruminants. In: JAPAN-BRASIL SYMPOSIUM ON SCIENCE AND TECNHOLOGY, 3., 1982, Tokyo. Proceeding... Tokyo: Agency of Industrial Science and Technology., out. p.25-27, 1982.
JANSON, J. Note explicative dês objects exposés par l'Ecole Agricole et Forestére de Komaba. Paris, 1889.

KONO, I.; FUKUYOSHI, S. Studies on the existence of eggs of pancreas flukes in the wall of the pancreatic duct and its significance. Bulletin of the Faculty of Agriculture, Kagoshima, v.30, p.111-116, 1966.

KONO, I.; SAKAMOTO, T.; YASUDA, N.; KITANO, Y.; TOSOE, T.; YAMAMOTO, Y. Pathological studies on the cattle heavily infected with Eurytrema coelomaticum. Bulletin of the Faculty of Agriculture, Kagoshima, v.30, p.111-116, 1980.

KUKHARENKO, N. S. Looking for antihelminthics against eurytrema infection in ruminants. In Glavneishie Gel'minty Sel'skonozyaistvennykh Shivotnykl Dal'nego vostoka i bor'ba s vimi (sbornik nauchnykh Trudov). Blagoveshchensk: [s.n], 1971.p. 20-22

LAPAGE, G. Monning's veterinary helminthology and entomology. 4. ed. London: Bailliére-Tindall \& Cox, Covent Garden, 1956.

LOOSS, A.; CUFFEY, E. On some parasites in the museum of the school of Tropical Medicine, Liverpool with a contribution on the case of distomasis of the liver and rectum. Annals of Tropical Medicine and Parasitology, London, v.1, p.123-154, 1907.

MANNINGER, R.; MÓCSY, J. Patologia y terapêutica especiales de los animales domésticos. 3. ed. Barcelona: Labor, 1973. v.2.

MARTIN, O. C. The incidence of Eurytrema pancreaticum in dairy cattle at the Dtri farm. Philippine Journal of Science, Manila, v.56, p.25-34, 1972.

MATTOS JÚNIOR, D. G. Eurytrema coelomaticum (Giard \& Billet, 1892), alguns aspectos do seu relacionamento com hospedeiros intermediários e definitivos sob condições experimentais. 1987. Dissertação (Mestrado)Universidade Federal Rural do Rio de janeiro, Rio de Janeiro.

MATTOS JÚNIOR, D. G.; VIANNA, S. S., O Eurytrema coelomaticum (Trematoda: Dicrocoeliidae) no Brasil. Arquivos Fluminenses de Medicina Veterinaria, Rio de Janeiro, v.2, n.1, p.3-7, 1987.

MAYAUDON, T. H. Eurytrema pancreaticum (Giard \& billet, 1892). Su presencia em bovinos (Bos taurus) de Venezuela. Revista de Medicina Veterinaria y Parasitologia, Caracas, v.13, p.175-176, 1954.

MORIYAMA, N.; TSUJI, M.; SETO, T. Karyological studies on bovine pancreatic flukes (Eurytrema sp.) and their phenotypes. Japanese Journal of Parasitology, Tokyo, v.29, n.4, p.281-291, 1980. 
NIEBERLE, K.; COHRS, P. Anatomia patológica especial dos animais domésticos. Lisboa: Fund. Calous. Gulbenkian, 1970.

NOSAKA, D.; ASHIZAWA, H.; NAGATA, Y. Pathological studies on bovine eurytrematosis. IV anthelmintic experiments and pathological findings of cows infected with Eurytrema species. Bulletin of the Faculty of Agriculture Miyazaki University, Miyazaki, v.17, p.104-132, 1970.

OGUNRINADE, A.; ADEGOKE, G. O. Bovine fasciolosis in Nigéria intercorrent parasitc and bacterial infections. Tropical Animal Health and Production, Edinburgh, v.14, n.2,p.121-124, 1982.

OLIVEIRA, O. C.; BECHARA, G. H. Aspectos microscópicos do pâncreas de bovino parasitado Eurytrema coelomaticum.. Arquivos de Biologia e Tecnologia, Curitiba, v.31, n.2, p.275-279, 1988.

PALATKIN, K. A. The economic loss caused by Eurytrema infection to animal farming. Helminthological Abstracts, Farnham Royal, v.47, n.8, p.344-345, 1978.

PINTO, C; ALMEIDA, A. J. Sinopse dos helmintos dos animais domésticos do Brasil. O Campo, Rio de Janeiro, v.6, p.60, 1935.

RAGUSA, A. L.; CAMPOS, M. S. Identificação de hospedeiro intermediário de Eurytrema coelomaticum (Giard e Billet, 1882) LOOSS: 1907, (Trematoda Dicrocoeliidae) em fazendas de criação de bovinos no estado de São Paulo - Brasil. Revista da Faculdade de Medicina Veterinária e Zootecnia da Universidade de São Paulo, São Paulo, v.13, n.1, p.269-287, 1976.

RIBEIRO, O. C.; BIANCHIN, I.; MELO, H. J. H.; GOMES, A.; PEREIRA, A. S. Lesões anátomo-patológicas observadas em infecções por Eurytrema pancreaticum em bovinos. In: CONGRESSO BRASILEIRO DE MEDICINA VETERINÁRIA, 17., 1980, Fortaleza. Anais... Fortaleza, 1980.

SAKAMOTO, H.; TASHIRO, T.; WATANABE, S.; SAKAMOTO, T.; KONO, I.; YASUDA, N. Clinicopathological findings of cattle infected with Eurytrema coelomaticum. Bulletin of the Faculty of Agriculture Kagoshima, Kagoshima, v.30, p.117-122, 1980.

SAKAMOTO, T.; KONO, I.; YASUDA, N.; YAMAMOTO, Y.; NAKAGAWA, H. Studies on Eurytrema coelomaticum. IV Comparison between anthelmintic effects of drug against Eurytrema coelomaticum in vitro and in vivo. Journal of the Faculty of Agriculture Iwate University, Tokyo, v.17, n.2, p.211-222, 1984.

SANTOS, I. F.; PRATA, L. F. Monitoramento de afecções crônica no abate de bovinos: ocorrência e distribuição geográfica de Eurytrema ssp. Em animais abatidos em Barretos, estado de São Paulo. Ars Veterinaria, Jaboticabal, v.14, n.1, p.61-69, 1998.
SEIFRIED, O. Curso de histopatologia para veterinarios y estudiantes. Barcelona: Labor, 1936.

SHIEN, Y. S.; LIU, L. L.; HUANG, S. W. Studies on eurytremiasis. II Pathological study of the pancreas of the cattle and goats naturally infected with Eurytrema pancreaticum. Journal / Chinese Society Veterinary Science, Taipei, v.5, n.2, p.133-138, 1979.

SOULSBY, E. J. L. Helminths, arthropods and protozoa of domesticated animals. 7. ed. Philadelphia: Lea \& Fabiger, 1982.

TANG, Z.; TANG, C. The biology and epidemiology of Eurytrema coelomaticum (GIARD; BILLET, 1892) and Eurytrema pancreaticum (JANSON, 1889) in cattle and sheep in China. Acta Zoologica, Stockholm, v.23, p.267282, 1977.

TORRES, C. M.; PINTO, C. Processos patogênicos determinados pelos trematodes Eurytrema fastosum e E. coelomaticum. Memorias do Instituto Oswaldo Cruz, Rio de Janeiro, v.31, n.4, p.731-746, 1936.

TRAVASSOS, L. Revisão da família Dicrocoeliidiae, Odhner, 1910. Rio de Janeiro: Imprensa Nacional, 1944. (Monografia do Instituto Oswaldo Cruz, n. 2)

UENO, H.; WANATABE, S. Curso de especialização em epidemiologia das nematodíases de ruminantes. Realizado no Depart. de Parasit. da USP. Comunicação Pessoal. 1968. As comunicações pessoais não vão em referencia, somente em notas de rodapé.

VIANNA, S. S. S. Técnica coproscópica de sedimentação para concentração de ovos de Eurytrema, Looss, 1907. 1984. Dissertação (Mestrado)- Universidade de São Paulo.

YAMAGUTI, S. A synoptical review of life histories of digenetic of vertebrates. Tokyo: Keikan Publish Co. Japan, 1975.

YAMAMURA, M. H. Algumas avaliações sobre a patologia e controle da euritrematose bovina. 1989. Tese (Doutorado em Medicina Veterinária, Parasitologia Veterinária Concentração Helmintologia Veterinária) - Universidade Federal Rural do Rio de Janeiro, Rio de Janeiro.

YAMAMURA, M. H.; HORNER, M. R.; LOPES, C. W. G. Avaliação patológica da Euritrematose em bovinos naturalmente infectados na região de Londrina, Paraná. Semina: ciências Agrárias, Londrina, v.16, n.1,p.89-99, mar. 1995.

YAMAMURA, M.H. Comunicação pessoal. 2005. (somente em nota de rodapé.... tem que ter o tema o dia que foi feita a comunicação

ZIEGLER, J. C. S.; VIDOTTO, O.; MARQUES, F. A. C.; ROCHA, M. A.; YAMURA, M. H.; VILLAS BOAS, L. L. Prevalência de Eurytrema coelomaticum em bovinos abatidos em Londrina, procedentes de diferentes microrregiões do estado do Paraná, Brasil. In: ENCONTRO DE PESQUISAS VETERINÁRIAS, 1., Londrina, 1984. Resumos... Londrina: UEL, 1984. 
